Kroneman, M., Essen, G.A. van, Paget, J.

Influenza vaccination coverage and reasons to refrain among high-risk persons in four European countries.

Vaccine: 24, 2006, p. 622-628

Postprint Version 1.0

Journal website http://www.sciencedirect.com/science/journal/0264410X

Pubmed link http://www.ncbi.nlm.nih.gov/entrez/query.fcgi?cmd=Retrieve\&db=pubmed\&dop t=Abstract\&list_uids=16169638\&query_hl=4\&itool=pubmed_docsum

DOI 10.1016/j.vaccine.2005.08.040

\title{
Influenza vaccination coverage and reasons to refrain among high-risk persons in four European countries
}

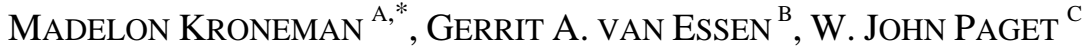 \\ a NIVEL, Netherlands Institute of Health Services Research, P.O. Box 1568, 3500 BN Utrecht, The \\ Netherlands \\ b European Scientific Working Group on Influenza (ESWI), Julius Center for Health Sciences and \\ Primary Care, University Medical Center Utrecht, P.O. Box 85060, 3508 AB Utrecht, The \\ Netherlands \\ c European Influenza Surveillance Scheme (EISS) Co-ordination Centre, NIVEL, P.O. Box 1568, 5300 \\ BN Utrecht, The Netherlands \\ * Corresponding author. Tel.: +31 30 2729640/700; fax: +31 30 2729729. E-mail address: \\ m.kroneman@nivel.nl (M. Kroneman).
}

\begin{abstract}
This paper examines influenza vaccine coverage using a population base of an average of 2300 persons in each of four European countries (Germany, Spain, Poland and Sweden). The reasons for non-vaccination of those in the high-risk groups were explored by questionnaire. The vaccine coverage rate (VCR) for elderly ranged from $18 \%$ in Poland to $67 \%$ in Spain. The VCR for high-risk population under 65 was $10 \%$ in Poland, $13 \%$ in Sweden, $27 \%$ in Germany and 30\% in Spain. The most important reasons mentioned for not being vaccinated were: perceived sufficient resistance to flu (33-42\%) in all countries; financial barriers in Poland (25\%), and not to qualify for the vaccination (30\%) and forgetfulness (20\%) in Germany. Receiving a personal invitation for the vaccination, results in higher coverage rates in all four countries. We think that future interventions could be directed towards: an information campaign with special attention to the high-risk groups due to disease; promotion of personal invitations; and, for Poland, solving financial barriers to vaccination.
\end{abstract}

\section{INTRODUCTION}

Influenza continues to be a considerable health problem of populations in Europe [1-3].

Complications due to influenza occur especially in elderly patients and patients with chronic conditions such as cardiovascular disorders and respiratory disorders [4,5]. Vaccination is an effective intervention, reducing mortality and morbidity as a result of influenza, especially in the elderly and patients with highrisk conditions [4,6-9]. Vaccine coverage rates (VCRs) in the elderly are not well documented in Europe even though it is relatively easy to define this target group; only 14 out of 26 European countries (53\%) were able to provide VCRs in the elderly during the 2000-2001 season [10]. The VCR among other high-risk patients is even more difficult to establish, a fact that is reflected in the very low number of countries in Europe that are able to produce these data (only 3-5 countries were able to provide these data for different groups at high risk due to disease) [10].

Most countries in Europe have the policy-intention to have persons at high risk vaccinated [11] and it is therefore important to know to what extent these target groups are indeed vaccinated. Within the countries that could provide coverage rates for the elderly in the European study mentioned before, a 
Kroneman, M., Essen, G.A. van, Paget, J.

Influenza vaccination coverage and reasons to refrain among high-risk persons in four European countries.

Vaccine: 24, 2006, p. 622-628

large variation in coverage rates was observed. This varied from under $25 \%$ for Romania and Finjand to over $80 \%$ for The Netherlands [10]. The WHO urges member states to have $50 \%$ of the high-risk population vaccinated in 2006 and 75\% in 2010. It is also important to know why the non-vaccinated in the high-risk population refrain from vaccination so that targeted policy measures can be taken. Reasons to refrain from vaccination may be personal considerations or a lack of information. Previous research showed that views about vaccine effectiveness and possible side effects [12-19] and views about the danger of and susceptibility for influenza $[12-14,20,21]$ influence vaccine coverage. Besides this, information provided by health professionals $[12,15,17-19,22]$, and especially personal invitations $[16,18,20,21,23,24]$, positively influence the coverage rates. Practical reasons like difficulty in coming to the medical clinic [19] or out-of-pocket payments compared to free-ofcharge vaccines may also play a role. Personal consideration may be hard to influence, however, providing information and lowering practical thresholds appear to influence VCRs positively.

This paper aims to gain insight into vaccine coverage among the different risk groups in four European countries and to provide a basis for targeted policy interventions to increase coverage rates.We therefore formulated the following research questions:

1. What is the influenza vaccination coverage rate of the healthy and high-risk population in four European countries?

2. What reasons do people in the high-risk groups have to refrain from influenza vaccination?

\section{METHODS}

\subsection{Choice of countries}

The study was carried out in Germany, Poland, Spain and Sweden. The choice of these countries was mainly based on practical reasons. We wanted to look at countries with poorly documented VCRs (especially for the high-risk population due to disease under 65 years of age), countries with decentralized health care systems (Germany, Spain, Sweden), and an Eastern European country. Besides, in none of these countries data were available on high-risk condition prevalence under 65 years of age [10]. Furthermore, all these countries recommend vaccination for the chronic conditions that were included in our study (e.g. cardiovascular diseases, lung diseases, diabetes mellitus, renal failure, and weakened immunity due to illness or treatment). The countries differ in co-payments for vaccination by in persons for whom vaccination is recommended. In Germany and Spain, the vaccination is free of charge. In Poland and in some regions in Sweden, co-payments are required. Since Poland has a much lower economic position compared to the other countries, we may expect that the out-of-pocket payments may form a more important barrier for vaccination uptake, especially compared to Sweden.

\subsection{Choice of data collection method}

VCRs in high-risk patients may be derived from GPinformation systems. However, this presupposes a fixed patient list, in order to have a valid population denominator. If no fixed patient list is available, the number of vaccinated high-risk population may be established, but not the population coverage rate (although indirect methods could be used to obtain an estimate). Earlier research in The Netherlands revealed that population surveys are a quick and reliable assessment method to study VCRs and establish population denominators [25]. In a population survey, the patient provides information about disease and vaccine coverage, while in a monitoring system, this information is obtained from medical professionals.

To establish the influenza VCRs, we used a questionnaire that contained questions about vaccine uptake, self-reported chronic conditions, reasons to refrain from vaccination, and whether one had received a personal invitation by a medical professional to have a vaccination. The questions were defined; no open-ended questions were included. For the selfreported chronic conditions, we asked whether these were confirmed by a physician. The questionnaire was validated in a study in The Netherlands during the 2000-2001 season [25]. The choice for 2500 is based on disease prevalence of at least $3 \%$ in the smallest of the three largest high-risk groups due to disease (based on prevalence data of The Netherlands, where diabetes (3.5\%) was the smallest group, after heart and lung diseases) [26], resulting in about 75 respondents and acceptable confidence intervals $( \pm 10 \%)$ for this group. The interviews in all four countries were held in March and April 2004. 
In Sweden $(n=2508)$, Germany $(n=2459)$ and Spain $(n=2505)$ a telephone survey was carried out. The survey was part of a national telephone-omnibus, carried out by an international market research organisation. A telephone-omnibus is an interview system that is carried out on a regular basis with a random selection of respondents, in which our questionnaire was included until on average 2500 respondents were interviewed. Because of this system, no relevant non-response data are available. In Poland, face-to-face interviews were held with 2000 respondents, using the same questionnaire, also included in a national omnibus. We would have preferred 2500 respondents in Poland as well, however, we could only choose 2000 or 3000 respondents, and 3000 respondents did not fit into the budget. For Poland, we were not able to choose the survey method. However, since the telephone density in Poland is about half of that in the other three countries, the face-to-face interview will probably provide a better random sample.

\subsection{Weighing the data}

In order to make the study population comparable with the real population of each country, weight factors were used. Since the omnibuses differ per country, resulting in different background variables that are collected, the variables used to compose the weight factor differ per country. For Germany, region, community size, age, gender, education, profession and household size were included. For Poland, community size, age, gender and household size were included. For Spain, region, age, gender and community size and for Sweden region, age and gender. The weight factors were constructed by the national bureaus that were responsible for the omnibuses. The data represented in this paper are the weighted data.

To compare our data with the national populations, we used the WHO European Health for All database, version June 2004, for mortality rates on diseases of the circulatory system and the respiratory system for the year 2001, and inpatient care admission rates for the year 2000 (Spain 1998).

\subsection{Operationalizations}

In this paper, the elderly are defined as those 65 years of age or older. One country's (Germany) definition for the target group 'elderly' showed a wider age group (60 years and older). However, for reasons of comparability,we use the definition 65 years and over for all countries. The high-risk group due to disease comprises those who report a chronic condition and are under 65 years of age. Urbanity was partitioned in three categories: low (less than 50,000 inhabitants), medium (50,000-500,000 inhabitants) and high (over 500,000 inhabitants). In the tables, the countries are presented in alphabetical order. In all countries, the respondents were aged 15 years and older.

\subsection{Statistical methods}

Differences among the countries were tested by means of Pearson's Chi-square test, a significance level of $p=0.05$ was set as criterion for significance. For calculation of confidence intervals we used Fleiss quadratic 95\% confidence intervals in the statistical package EpiInfo 6.

\section{RESULTS}

\subsection{Description of the samples}

Spain (10\%) and Sweden (13\%) had the lowest percentage of the high-risk population under 65 years of age with chronic diseases. In Germany (20\%) and Poland (21\%), almost twice as many persons reported belonging to this specific highrisk group. The high percentage of cardio vascular disease (13\%) in Poland is striking, as is the relatively high percentage of lung diseases in Germany (7\%) (see Table 1). The high percentage for cardio vascular disease is reflected in the high-standardized death rates for diseases (published in the WHO Health for All database) of the circulatory system (432 per 100,000 persons) and ischemic heart disease (133 per 100,000) in Poland. The Polish rates are, respectively, at least 250 per 100,000 and 10 per 100,000 higher compared to the three other countries. In Germany, about 24\% of the population was hospitalized in 2000 (WHO Health for All database), which is $8 \%$ more than the second highest (Sweden with 16\%). This may be an indication that there are indeed more chronic ill persons in Germany, compared to Sweden and Spain. 


\section{[ TABLE 1 ]}

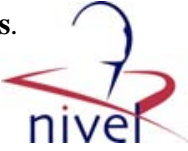

\subsection{Differences among the countries in VCRs}

The highest proportion of vaccinated elderly was found in Spain, where two out of three elderly persons were vaccinated (see Table 1). Poland showed the lowest VCR for elderly, only one out of five elderly persons received an influenza vaccination last winter. The highest proportion of vaccinated persons among those at high risk due to disease were also found in Spain and Germany. Sweden and Poland scored significantly lower. For the persons at high risk due to disease, we found a quite persistent pattern showing a higher coverage rate for cardiovascular and diabetes patients compared to lung patients and other diseases, although the difference is significant only for heart and lung disease in Germany. The highest proportion of vaccinated healthy persons was found in Germany, with one out of five vaccinated against flu. In contrast, the lowest proportion of vaccinated healthy persons was found in Sweden, with 1 out of 20. The high proportion of vaccinated healthy persons in Germany may be due to the fact that vaccination is recommended for those aged 60 and over in Germany. However, if we define the healthy persons as those under 60 years of age without a chronic condition, $19 \%$ were vaccinated in Germany and this continues to be the highest percentage among the four countries (Spain 11\%, Poland 9\%, Sweden 4\%).

\subsection{Reasons for not having a vaccination}

We divided the reasons for not having a vaccination for persons in the high-risk groups into misconceptions and perceived barriers. The most mentioned misconception is that one has sufficient resistance to flu. At least one out of three non-vaccinated in all four countries mentioned this reason (see Table 2). In Germany, another misconception is often mentioned: almost one out of three nonvaccinated high-risk persons think they do not qualify for the vaccination. Besides this, the opinion that flu is not a serious disease (14\%) played an important role in Germany. The Swedish nonvaccinated high-risk persons mentioned that they did not qualify for influenza vaccination (15\%) and that influenza is not a serious disease (12\%). In our study, we did not find differences in misconceptions among all four countries for gender, type of high-risk group (elderly or chronic ill under 65 years of age) and urbanity (data not shown).

\section{[ TABLE 2 ]}

Concerning the barriers, one perceived barrier played an important role for the Polish persons at high risk: the cost of the vaccination. One out of four persons that were not vaccinated mentioned this. This is especially the case for the low (25\%, 95\% CI: 20-31) and medium (29\%, 95\% CI: 23-43) urbanized areas, in the large cities, only $11 \%$ (95\% CI: 6-20) mentioned this barrier. Interestingly, the costs are hardly mentioned as a barrier by Swedish respondents, although in some of the counties a co-payment is required. In Germany, often mentioned barriers (by at least 1 out of 10 non-vaccinated high-risk persons) are forgetfulness (20\%), having had bad experiences in the past (15\%) and being against vaccination on principle (10\%). Elderly in Germany tend to forget vaccination less often (13\%, 95\% CI: 9-18) than chronic ill under the age of 65 (24\%, 95\% CI: 20-29). In Spain, having had bad experiences in the past (10\%) and being on principle against vaccination (9\%) were mentioned beside having enough resistance (38\%).

\subsection{Personal invitations}

When persons at high risk receive a personal invitation for having an influenza vaccination, the VCR was significantly higher in all four countries (Chi-square test significant at $p>0.00$ ). This is also the case in Poland, where the price of the vaccination is an often mentioned barrier (see Table 3). Only a few persons belonging to the high-risk groups received a personal invitation in Sweden. The highest proportion of invitations among high-risk persons was found in Germany (about one out of two).

\section{[ TABLE 3 ]}

\section{CONCLUSIONS AND DISCUSSION}

Our first research question concerned the coverage rates for influenza vaccination among the highrisk population in selected European countries. There were clear differences in VCRs in the four 
Kroneman, M., Essen, G.A. van, Paget, J.

Influenza vaccination coverage and reasons to refrain among high-risk persons in four European countries.

Vaccine: 24, 2006, p. 622-628

countries (Germany, Poland, Spain and Sweden). Spain showed the highest coverage rates for the high-risk groups due to age and disease, Poland displayed the lowest coverage rates for both groups. For the high-risk group due to disease in Poland, theVCRwas even equal to the healthy population's, indicating a non-targeted vaccination strategy. In all countries, the VCR of the elderly was significantly higher compared to high-risk persons due to disease, and in all countries, except Poland, the VCR for the high-risk group due to disease was higher than the VCR in the group of healthy persons under 65 years of age. This indicates that the recommendations for influenza vaccination are followed at least to some extent. The quite persistent pattern showing a higher VCR for cardiovascular and diabetes patients compared to lung patients and other diseases was also found in previous research in The Netherlands [25].

In all four countries, the VCRs were significantly lower compared to the coverage rates found in The Netherlands in earlier research (elderly 74\%, 95\% CI: 71-77, due to disease 53\%, 95\% CI: 48-58; this study used the same method) [25], which indicates that there is room for improvement.However, there is already some improvement compared to earlier years. In Spain, the VCR among the elderly increased from 51\% in 1997 [27] to 69\% in 2003-2004.

This brings us to our second research question, which dealt with reasons for not being vaccinated. In all countries, there is a large number of persons in the high-risk groups that have the opinion that their natural resistance provides sufficient protection against influenza (33-42\%). Although most people from this group acknowledge that influenza may be a serious illness, they do not think they themselves are susceptible to it. In Poland, financial barriers are perceived when having a vaccination. One out of four non-vaccinated high-risk group persons in Poland mentioned this as a reason for not having a vaccination. In Germany, people often forget to go for a vaccination (almost one out of four). In Sweden and Germany, 1 out of 10 believes that influenza is not a serious illness. Previous research revealed similar reasons for not having a vaccination $[19,21,28-30]$.

Future research could be directed towards practical barriers people experience in getting vaccinated. Maybe the procedure to be followed causes people to refrain from vaccination. For instance, there may be a difference in countries where patients can go for special vaccination sessions (maybe also in the evenings) compared to countries where the patients have to collect their own vaccine dose at the pharmacy and go the GP afterwards. At this moment there is little insight in the way this is organised among the different European countries.

For policy interventions, we would like to propose three different intervention options based on the results of our study. Firstly, in an information campaign, attention could be given to who qualify for vaccination and what is the risk of influenza and to what extent relying on nature resistance is a valid reason for not getting vaccinated. The information campaign should be targeted not only at those over 65 years of age, but especially at those belonging to the high-risk groups due to disease, since in these groups coverage rates are relatively low. A second intervention may be to personally invite the highrisk group for vaccination. In this study, we showed that having a personal invitation resulted in a significantly higher compliance in all countries, which is in accordance with earlier research [25,31]. The group of people that may be hard to convince will be those who refused a vaccination on principle. However, this group is quite small in all countries. A third option applies especially to Poland. Here, the financial barriers appear to represent an important obstacle. Policy measures to reduce the financial burden of vaccination for persons belonging to the high-risk groups would be beneficial. However, all of these proposed measures should be evaluated for their effectiveness.

This study has several limitations. One limitation of the questionnaire used is that belonging to a high-risk group is self-reported, although we asked whether a physician had confirmed the diagnosis. This may lead to over-reporting as well as under-reporting [32-34]. In this study, there may be overreporting of chronic conditions in Germany. German respondents reported 1.29 diseases per person, which is the highest among the four countries (Poland: 1.00, Spain: 1.13, Sweden: 1.19). Also, the fact that a relatively high proportion of people in Germany reported belonging to this high-risk group (20\%) in combination with the fact that an often-mentioned reason for non-compliance was "I do not qualify for influenza vaccination” (28\%) may be an indicator for over-reporting a chronic condition. Besides this, the German population reported more reasons for non-vaccination compared to the other three countries (Germany 1.66 per person, Poland: 1.11, Spain: 1.09, Sweden: 1.19). In Poland, this reason (not qualifying for influenza vaccination) was not frequently mentioned, which may be an indication that the health status of the Polish population is indeed worse compared to Sweden and 
Kroneman, M., Essen, G.A. van, Paget, J.

Influenza vaccination coverage and reasons to refrain among high-risk persons in four European countries.

Vaccine: 24, 2006, p. 622-628

Spain. A second limitation is the fact that the interviews were held in March and April. It would have been better to have a shorter period of time between the vaccination period and the survey. A third limitation is the different data collection method in Poland. The face-toface interviews may lead to a higher proportion of elderly and chronic ill in the sample, because these persons can be expected to be more often at home compared to healthy persons. It is not clear whether this method leads to more social desirable answers compared to the telephone interviews.

Summarizing, there is quite some variation among these countries, but none of the countries (except Spain for the elderly) already meet the WHO-target for 2010 of $75 \%$ of all high-risk population vaccinated. The WHO-target for 2006, 50\% of the high-risk population vaccinated, is met for the elderly for all countries except Poland. However, for the chronic ill none of the four countries meet this target. This implies that there is room for improvement. We would like to propose three future interventions: information campaigns paying special attention to the high-risk groups due to disease; promotion of personal invitations; and, especially for Poland, the removal of financial barriers for high-risk group members.

\section{ACKNOWLEDGEMENT}

This study was funded by the European Working Group on Influenza (ESWI).

\section{REFERENCES}

[1] Snacken R. Control of influenza. Public health policies. Vaccine 1999;17(Suppl. 3):S61-3.

[2] Tacken M, De Bakker D, Verheij R, Mulder J, Van den Hoogen H, Braspenning J. Evaluatie griepvaccinatiecampagne 2001 (evaluation influenza vaccination campaign 2001). Nivel: Utrecht; 2002.

[3] Szucs T, Behrens M, Volmer T. Public health costs of influenza in Germany 1996-a cost-of-illness analysis. Med Klin 2001;96(2): 63-70.

[4] Monto AS. Preventing influenza in healthy adults: the evolving story. J Am Med Assoc 2000;284(13):1699-701.

[5] McDaid D, Maynard A. Translating evidence into practice: the case of influenza vaccination. Eur J Public Health 2001;11(4):453- 5.

[6] Monto AS. The clinical efficacy of influenza vaccination. Pharmacoeconomics 1996;(Suppl. 3):1622.

[7] Christenson B, Lundbergh P. Comparison between cohorts vaccinated and unvaccinated against influenza and pneumococcal infection. Epidemiol Infect 2002;129(3):515-24.

[8] Gross PA, Hermogenes AW, Sacks HS, Lau J, Levandowski RA. The efficacy of influenza vaccine in elderly persons. A meta-analysis and review of the literature. Ann Intern Med 1995;123(7):51827.

[9] Van Essen GA, Sorgdrager YCG, Salemink GW, Govaert ThME, Van den Hoogen JPH, Van der Laan JR. In: Thomas S, Geijer RMM, Van der Laan JR, Wiersma Tj, editors. NHG standaarden voor de huisarts, deel II (Netherlands GP-association: standards for the GP, part II). Utrecht: Bunge; 1996; NHG-standaard influenza en influenzavaccinatie (Netherlands GP-association's standard influenza and influenza vaccination), p. 179-87.

[10] Kroneman M, Paget WJ, Van Essen GA. Influenza vaccination in Europe: an inventory of strategies to reach target populations and optimise vaccination uptake. Eurosurveillance 2003;8(6):130-8.

[11] Van Essen GA, Palache AM, Forleo E, Fedson DS. Influenza vaccination in 2000: recommendations and vaccine use in 50 developed and rapidly developing countries. Vaccine 2003;21:1780-5.

[12] Evans MR, Watson PA. Why do older people not get immunised against influenza? A community survey. Vaccine 2003;21 (19-20):2421-7.

[13] Bedford D, Howell F, Corcoran R. The provision of influenza vaccine to patients over 65 years by general practitioners. Ir Med J 1997;90(6):231-2 [JID - 0430275].

[14] Cornford CS, Morgan M. Elderly people's beliefs about influenza vaccination. Br J Gen Pract 1999;49(441):281-4 [JID - 9005323].

[15] Armstrong K, Berlin M, Schwartz JS, Propert K, Ubel PA. Barriers to influenza immunization in a low-income urban population. Am J Prev Med 2001;20(1):21-5.

[16] Gosney M. Factors affecting influenza vaccination rates in older people admitted to hospital with acute medical problems. J Adv Nurs 2000;32(4):892-7 [JID - 7609811]. 
Kroneman, M., Essen, G.A. van, Paget, J.

Influenza vaccination coverage and reasons to refrain among high-risk persons in four European countries.

Vaccine: 24, 2006, p. 622-628

[17] Lewis-Parmar H, McCann R. Achieving national influenza vaccine targets-an investigation of the factors affecting influenza vaccine uptake in older people and people with diabetes. Commun Dis Public Health 2002;5(2):119-26.

[18] Nguyen-Van-Tam JS, Nicholson KG. Influenza immunization; vaccine offer, request and uptake in high-risk patients during the 1991/2 season. Epidemiol Infect 1993;111(2):347-55.

[19] Nichol KL, Lofgren RP, Gapinski J. Influenza vaccination. Knowledge, attitudes, and behavior among high-risk outpatients. Arch Intern Med 1992;152(1):106-10.

[20] Hutchinson HL, Norman LA. Compliance with influenza immunization: a survey of high-risk patients at a family medicine clinic. J Am Board Fam Pract 1995;8(6):448-51.

[21] Van Essen GA, Kuyvenhoven MM, de Melker RA. Why do healthy elderly people fail to comply with influenza vaccination? Age Ageing 1997;26(4):275-9.

[22] Watkins $\mathrm{J}$. Effectiveness of influenza vaccination policy at targeting patients at high risk of complications during winter: cross sectional survey. BMJ 1997;315(7115):1069-70 [JID - 8900488].

[23] Furey A, Robinson E, Young Y. Improving influenza immunisation coverage in 2000-2001: a baseline survey, review of the evidence and sharing of best practice. Commun Dis Public Health 2001;4(3):183-7.

[24] Igoe G, Bedford D, Howell F, Collins S. How to improve the uptake of influenza vaccination in older persons at risk. Ir J Med Sci 1999;168(2):107-8.

[25] Kroneman MW, Van Essen GA, Tacken MAJB, Paget WJ, Verheij R. Does a population survey provide reliable influenza vaccine uptake rates among high-risk groups? A case study of The Netherlands. Vaccine 2004;22(17-18):2163-70.

[26] Tacken M, Verheij R, Mulder J, Van den Hoogen H, Braspenning J. Monitoring griepvaccinatiecampagne 2003 (monitoring the influenza vaccination campaign 2003). Nivel: Utrecht; 2004.

[27] Sarria-Santamera A, Timoner J. Influenza vaccination in old adults in Spain. Eur J Public Health 2003;13(2):133-7.

[28] Cornford CS, Morgan M. Elderly people's beliefs about influenza vaccination. $\mathrm{Br} \mathrm{J}$ Gen Pract 1999;49(441):281-4.

[29] Mulet Pons MJ, Sarrion Ferre MT, Barea MA, Marin RN, Blanquer Gregori JJ, Melchor Penella MA. Evaluation of the completion of influenza vaccination. Aten Primaria 1995;16(7):423-7.

[30] Telford R, Rogers A. What influences elderly peoples' decisions about whether to accept the influenza vaccination? A qualitative study. Health Educ Res 2003;18(6):743-53.

[31] Hull S, Hagdrup N, Hart B, Griffiths C, Hennessy E. Boosting uptake of influenza immunisation: a randomised controlled trial of telephone appointing in general practice. $\mathrm{Br} \mathrm{J}$ Gen Pract 2002;52(482): 712-6.

[32] Martin LM, Leff M, Calonge N, Garrett C, Nelson DE. Validation of self-reported chronic conditions and health services in a managed care population. Am J Prev Med 2000;18(3):215-8.

[33] Lampe FC, Walker M, Lennon LT, Whincup PH, Ebrahim S. Validity of a self-reported history of doctor-diagnosed angina. J Clin Epidemiol 1999;52(1):73-81.

[34] Sheridan CL, Mulhern M, Martin D. Validation of a self-report measure of somatic health. Psychol Rep 1998;82(2):679-87. 
Kroneman, M., Essen, G.A. van, Paget, J.

Influenza vaccination coverage and reasons to refrain among high-risk persons in four European countries.

Vaccine: 24, 2006, p. 622-628

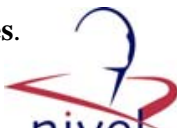

\section{TABLES}

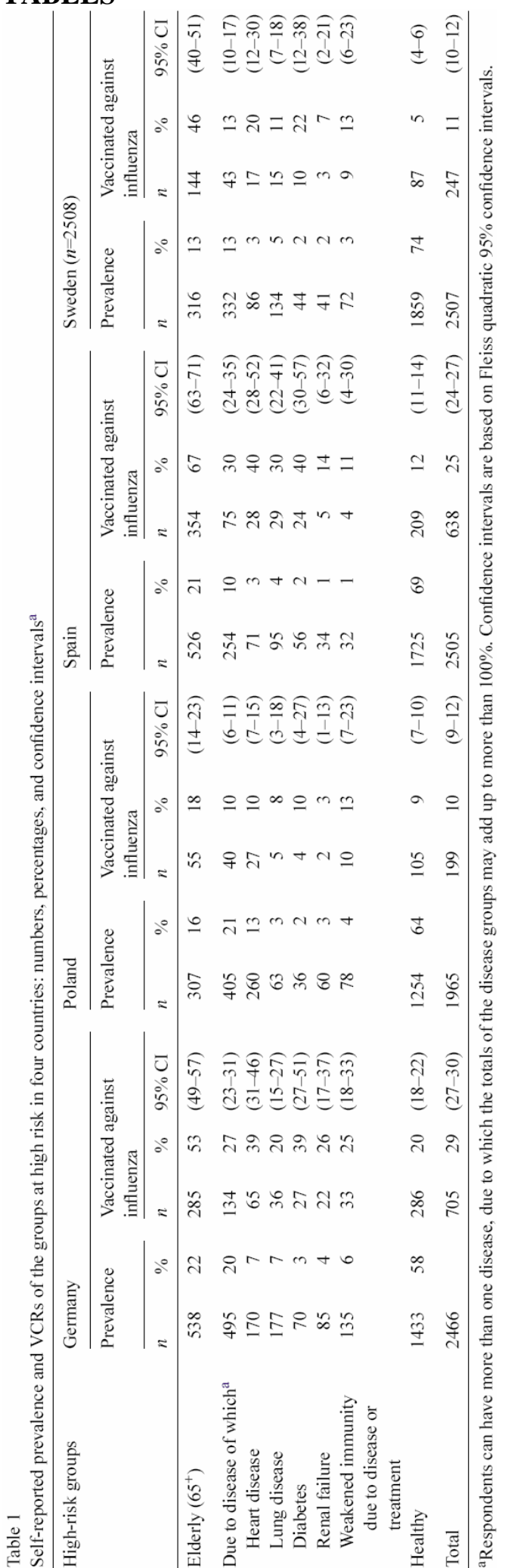


Kroneman, M., Essen, G.A. van, Paget, J.

Influenza vaccination coverage and reasons to refrain among high-risk persons in four European countries.

Vaccine: 24, 2006, p. 622-628

Table 2

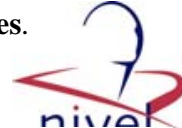

Reasons to refrain from vaccination per country for high-risk persons (elderly and high risk due to disease combined)

\begin{tabular}{|c|c|c|c|c|c|c|c|c|}
\hline \multirow[t]{2}{*}{ Reasons to refrain from vaccination ${ }^{\mathrm{a}}$} & \multicolumn{2}{|c|}{ Germany $(n=614)$} & \multicolumn{2}{|c|}{ Poland $(n=617)$} & \multicolumn{2}{|c|}{ Spain $(n=351)$} & \multicolumn{2}{|c|}{ Sweden $(n=461)$} \\
\hline & $n$ & $\%$ & $n$ & $\%$ & $n$ & $\%$ & $n$ & $\%$ \\
\hline \multicolumn{9}{|l|}{ Misconceptions } \\
\hline I have sufficient resistance to flu & 254 & 42 & 215 & 35 & 132 & 38 & 151 & 33 \\
\hline I do not qualify for influenza vaccination & 185 & 30 & 42 & 7 & 22 & 6 & 67 & 15 \\
\hline Influenza is not a serious illness & 83 & 14 & 29 & 5 & 8 & 2 & 57 & 12 \\
\hline \multicolumn{9}{|l|}{ Barriers } \\
\hline The vaccination is too expensive & 28 & 5 & 152 & 25 & 0 & 0 & 10 & 2 \\
\hline It slipped my mind & 120 & 20 & 47 & 8 & 22 & 6 & 35 & 8 \\
\hline I was unable to attend at the given time & 30 & 5 & 4 & 1 & 7 & 2 & 12 & 3 \\
\hline I have had bad experiences with flu & 89 & 15 & 19 & 3 & 36 & 10 & 28 & 6 \\
\hline On principle, I am against vaccination & 68 & 11 & 42 & 7 & 31 & 9 & 30 & 7 \\
\hline $\begin{array}{l}\text { The General Practitioner or public health } \\
\text { authority carrying out the flu vaccination } \\
\text { was too far away for me }\end{array}$ & 20 & 3 & 12 & 2 & 1 & 0 & 7 & 1 \\
\hline \multicolumn{9}{|l|}{ Miscellaneous } \\
\hline My physician considered it unnecessary & 51 & 8 & 16 & 3 & 20 & 6 & 12 & 3 \\
\hline Other & 75 & 12 & 90 & 15 & 101 & 29 & 125 & 27 \\
\hline Don't know & 17 & 3 & 15 & 2 & 4 & 1 & 16 & 4 \\
\hline
\end{tabular}

${ }^{a}$ Respondents were allowed to state more than one reason, thus individual percentages may add up to more than $100 \%$.

Table 3

Personal invitation for influenza vaccination in the high-risk population (both due to age and disease) and vaccination coverage rates for the population at high risk who $\mathrm{did} / \mathrm{did}$ not receive a personal invitation

\begin{tabular}{llll}
\hline & Germany & Poland & Spain \\
\hline Received personal invitation, $n(\%)^{\text {a }}$ & $558(52 \%)$ & $191(27 \%)$ & $288(37 \%)$ \\
Vaccination coverage within group with personal invitation (\%) $_{\text {Vaccination coverage within group without personal invitation (\%) }}$ & 62 & 35 & 71 \\
\hline
\end{tabular}

a Percentage of total population at high risk. 